\title{
Les prénoms et l'image des filles
}

Recherches sur les prénoms féminins en Ile-de-France autour de la période révolutionnaire (1775-1825)

\section{Philippe Daumas}

\section{(2) OpenEdition \\ 1 Journals}

Édition électronique

URL : https://journals.openedition.org/ahrf/130

DOI : $10.4000 /$ ahrf. 130

ISSN : 1952-403X

Éditeur :

Armand Colin, Société des études robespierristes

\section{Édition imprimée}

Date de publication : 1 décembre 2000

Pagination : 111-129

ISSN : 0003-4436

\section{Référence électronique}

Philippe Daumas, "Les prénoms et l'image des filles », Annales historiques de la Révolution française [En ligne], 322 I octobre-décembre 2000, mis en ligne le 02 mai 2007, consulté le 23 avril 2022. URL : http://journals.openedition.org/ahrf/130 ; DOI : https://doi.org/10.4000/ahrf.130

Ce document a été généré automatiquement le 23 avril 2022.

Tous droits réservés 


\title{
Les prénoms et l'image des filles
}

\author{
Recherches sur les prénoms féminins en Ile-de-France autour de la \\ période révolutionnaire (1775-1825)
}

\section{Philippe Daumas}

1 Le choix d'un prénom n'a jamais été un acte gratuit. Pendant des siècles, il a été fortement déterminé par des contraintes sociales extrêmement pesantes rites de passage, relation entre la transmission du prénom et le lien personnel, emprise de la religion sur la vie familiale. Face au poids des traditions, la part de liberté laissée à l'individu paraissait alors bien réduite, elle-même conditionnée par des phénomènes moins perceptibles, moins aisément mesurables, mais tout aussi déterminants réseaux de relations, systèmes de références idéologiques ou culturelles, phénomènes de mode...

2 Cependant, l'étude des prénoms est aujourd'hui considérée comme un indicateur culturel particulièrement pertinent, dans la mesure où elle met en évidence aussi bien les conditions sociales, religieuses ou idéologiques influant sur les choix personnels, que les réponses des individus ou des groupes sociaux à ces pressions et à ces sollicitations. Pour la période révolutionnaire, de récentes études ont montré qu'audelà du phénomène des "prénoms républicains, objet de passionnantes recherches mais très limité dans le temps et dans l'espace, une étude portant sur l'ensemble des prénoms pouvait s'avérer très riche d'enseignements ${ }^{1}$.

3 La présente contribution propose un exemple - parmi beaucoup d'autres - de piste de recherche faire apparaître, à travers l'étude des prénoms féminins attribués pendant cinquante années englobant la Révolution française, les conditions spécifiques du choix de ces prénoms, les attitudes parentales qu'elles révèlent à l'égard des filles, témoignant à leur manière d'une image ou même d'un statut de la féminité observer enfin l'évolution de ces attitudes, tout au long d'une période marquée par un événement d'une force exceptionnelle où la rencontre du "temps court et du "temps long détermine d'importants changements sociaux et culturels.

Méthodologie d'une enquête

4 Les matériaux de la présente recherche sont tirés d'une thèse en cours sur les comportements familiaux dans les campagnes d'île-de-France autour de la période 
révolutionnaire. Le relevé systématique des naissances et des baptêmes entre 1775 et 1825, dans onze villages ${ }^{2}$ représentant une population d'environ 7000 habitants, a fourni un corpus général de 10863 naissances, 9545 baptêmes, 22227 prénoms attribués, et pour les filles de 5417naissances, 4759 baptêmes, 11400 prénoms attribués. Pour améliorer la fiabilité des statistiques et la lisibilité des graphiques, les données quantitatives ont été traitées par périodes de cinq ans

Les onze villages ont été choisis en fonction de la disponibilité des sources, mais aussi de manière à présenter la plus large diversité de caractéristiques taille, population, activités économiques, proximité de Paris. S'ils se différencient fortement par leurs réactions face à l'événement révolutionnaire, les uns connaissant une forte activité politique, d'autres plus indifférents, d'autres enfin marqués par des clivages importants et des affrontements, ils ont en commun d'avoir été confrontés à un contexte économique très difficile, marqué par les mauvaises récoltes et les hivers rigoureux, aggravé par la crise monétaire, les levées d'hommes et les réquisitions, plus lourdes en Île-de-France que partout ailleurs.

6 Ils ont enfin en commun un caractère déterminant l'influence de la capitale, résultant non seulement de la proximité géographique mais aussi des relations économiques et humaines, facilitant la circulation des informations, la diffusion des écrits, donc celle des idées. Cette proximité confère sans doute à la vie politique, sociale et culturelle des campagnes d'île-de-France une profonde originalité.

7 Toute étude sérieuse sur l'ensemble des prénoms exige une mise en relation de l'état civil avec les registres de catholicité. Les actes de baptême fournissent en effet de précieuses indications sur les parrains et marraines, indispensables à l'étude des critères de choix et des modes de transmission. Si l'enquête repose essentiellement sur des statistiques de lecture apparemment simple, l'analyse en est souvent incertaine et pose d'importants problèmes méthodologiques. La motivation du choix individuel étant par définition insaisissable, le nombre est pour le chercheur la seule garantie de fiabilité. Les difficultés d'interprétation exigent de croiser les informations fournies par les différents indicateurs.

8 Les études publiées sur les prénoms depuis quelques années ont montré les difficultés des chercheurs à s'accorder sur la définition claire d'objectifs et de méthodes d'analyse, et même sur le vocabulaire à utiliser... Dans le souci d'écarter la notion de prénom composé, anachronique à l'époque étudiée et source d'ambiguïtés, le mot prénom ne désigne ici que l'unité prénominale, le mot prénomination qualifiant l'ensemble des prénoms reçus par un enfant. Bien évidemment, la dissociation des prénoms attribués pour certaines recherches ne fait en rien obstacle à une enquête approfondie sur les associations de prénoms, indispensable notamment pour l'étude des prénoms révolutionnaires, ou encore pour la mise en évidence de "formules de prénomination consacrées par l'usage.

9 Il convient enfin de fixer avec modestie les limites d'une telle enquête, en soulignant l'importance des lacunes et des incertitudes liées aux sources. On sait par expérience que les taux d'erreurs et d'omissions dans les actes sont loin d'être négligeables. On doit admettre par ailleurs, dans une proportion impossible à déterminer, l'existence de critères de choix des prénoms intraduisibles dans les actes emprunts à des membres de la famille autres que parents et parrains (notamment aux grands-parents) ou à des personnes extérieures à la famille, autres motivations relevant de relations personnelles ou d'obligations morales. Rappelons enfin combien il est frustrant de ne 
pouvoir cerner avec précision la notion de prénom usuel et son décalage évident avec la prénomination officielle, de l'état civil comme du baptême. L'emprunt d'un prénom à un parent ou à un parrain a-t-il la même signification s'il s'agit de son prénom usuel ou d'un prénom qu'il n'utilise pas La recherche des prénoms usuels se heurte à des difficultés méthodologiques insurmontables recours à des actes où les personnes sont citées comme intervenants secondaires, ou à des archives autres que celles de l'état civil, importance de l'homonymie à cette époque, nécessité d'une reconstitution des familles matériellement irréalisable à grande échelle. Il faut donc accepter a priori l'idée d'un décalage entre la réalité vécue et l'image que nous en donnent les actes. Si les données chiffrées sont clairement observables, leur analyse ou leur interprétation exigent beaucoup de prudence et ne peuvent donner lieu, en tout état de cause, qu'à des hypothèses.

L'évolution du corpus : diversité, féminisationÉvolution du stock

Un des faits marquants de la période est l'augmentation considérable du stock de prénoms utilisés. Le graphique ci-dessous montre que si le phénomène est général, il touche beaucoup plus fortement les prénoms féminins 76 prénoms utilisés en 1775/1779 et 163 prénoms en 1820/1825, soit une augmentation de 114\% Dans le même temps, le stock des prénoms masculins n'augmente que de $47 \%$. La progression est constante et assez régulière tout au long de la période, avec une légère pointe en 1790/1794, moins marquée que pour les prénoms masculins, ceux-ci ayant été davantage affectés par la vague des prénoms révolutionnaires. À partir de 1795/1799, le stock des prénoms féminins devient supérieur à celui des prénoms masculins, et le restera jusqu'à la fin de la période étudiée.

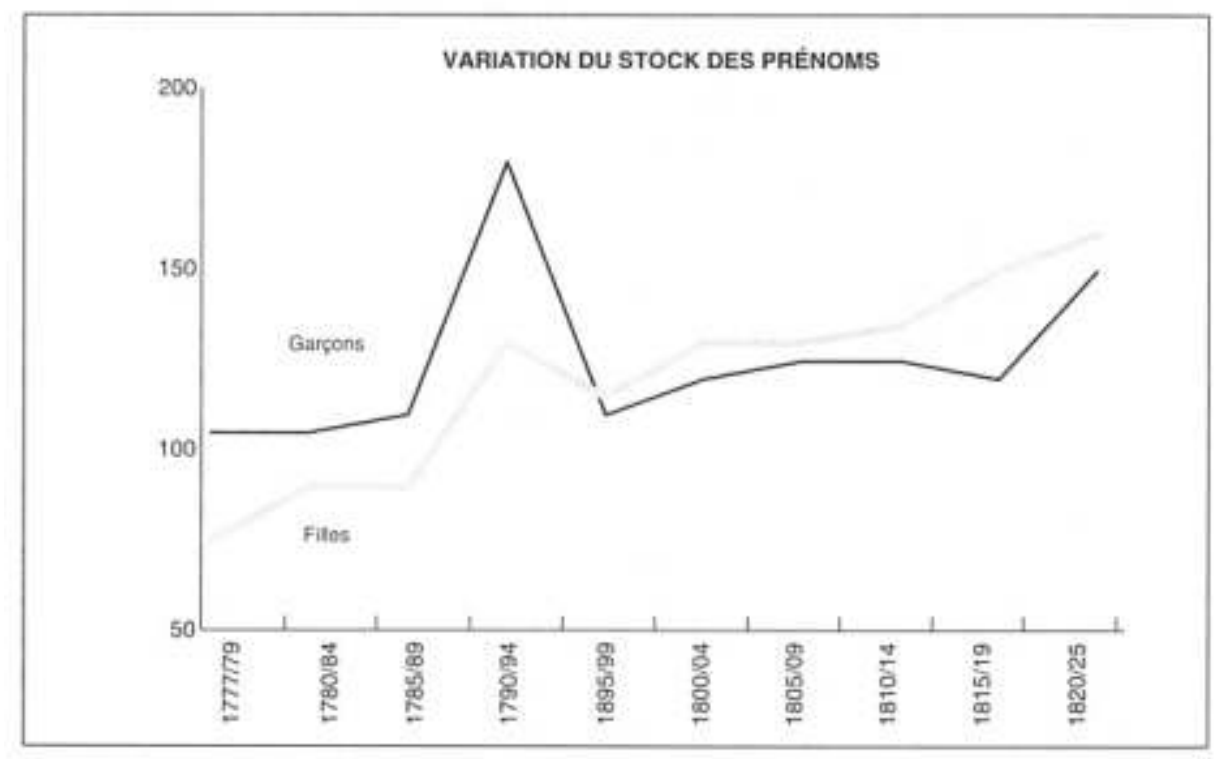

11 L'étude de la composition du stock des prénoms met clairement en évidence les mécanismes de cette évolution. À partir des années 1790/1794, compte non tenu des prénoms révolutionnaires, 82prénoms nouveaux apparaissent à raison d'une dizaine environ par période de cinqans, avec deux pointes en 1795/1799 (17prénoms nouveaux) et en 1810/1814 (13prénoms nouveaux). Dans le même temps, on ne relève que 10disparitions de prénoms par rapport au stock d'origine. L'annexe1 présente la liste des prénoms concernés, avec leur date d'apparition ou de disparition. 

momentanément inutilisés (Adèle, Lucie), une large majorité apparaissent comme de véritables innovations et présentent des caractères originaux. On voit ainsi apparaître en tant que prénoms d'anciens diminutifs, tels que Jeannette, Annette, Nanette... Les prénoms empruntés aux personnages de la littérature ou du théâtre (Virginie, Héloïse, Zoé) se multiplient et surtout s'implantent dans les milieux les plus modestes familles de vignerons, de journaliers, familles d'ouvriers à Ivry. Dans le même temps se développe le goût de l'originalité esthétique (Lussine, Flore, Athénaïse), parfois enrichie d'une note d'exotisme (Mélina, Zélia, Zulma). Les variantes se multiplient à partir du prénom Élisabeth apparaissent Élisa, Élise, Lisa, Lise à partir de Céline, on trouve Célina et Célinie...

Mais le phénomène dominant est la création de prénoms féminins à partir de prénoms masculins déjà existants, par simple rajout d'un suffixe (ie, ine, ette). Ainsi se multiplient d'un prénom envisagé d'un point de vue strictement phonétique, sans aucun égard pour sa signification. Cette catégorie représente $40 \%$ de l'ensemble des nouveautés entre 1790 et 1825 . Ces prénoms, tous apparus pendant et après la Révolution, sont étrangers au martyrologe chrétien. Ils sont, tout comme les prénoms révolutionnaires, à l'origine d'un phénomène sur lequel nous reviendrons le refus de l'Église, source de différences entre les prénoms de baptême et ceux de l'état civil.

Les disparitions de prénoms féminins sont peu nombreuses durant la période étudiée. Certaines sont bien sûr temporaires et liées à des phénomènes de mode (Colombe, Opportune par exemple). Il est toutefois significatif que sept de ces disparitions sur dix concernent des prénoms masculins. La relation est évidente avec la multiplication des féminisations de prénoms masculins. Sur l'ensemble de la période, l'attribution de prénoms masculins à des filles tend à se raréfier elles représentent $3 \%$ des prénominations avant la Révolution, et seulement $0,7 \%$ en 1820/1825. Le phénomène témoigne peut-être, bien que modestement, de la reconnaissance accrue d'une identité féminine.

Formes et sonorités

15 La forme et la sonorité des prénoms féminins connaissent également en cinquanteans des évolutions importantes. On observe tout d'abord une tendance à l'allongement, par la multiplication des prénoms trisyllabiques ou quadrisyllabiques. Ceux-ci représentent $32 \%$ des prénoms attribués en $1775 / 1779$, et passent à $44 \%$ en $1820 / 1825$. Le graphique ci-dessous fait apparaître un écart considérable avec les prénoms masculins, qui ne passent dans le même temps que de 4 à $14 \%$ des prénoms attribués. 


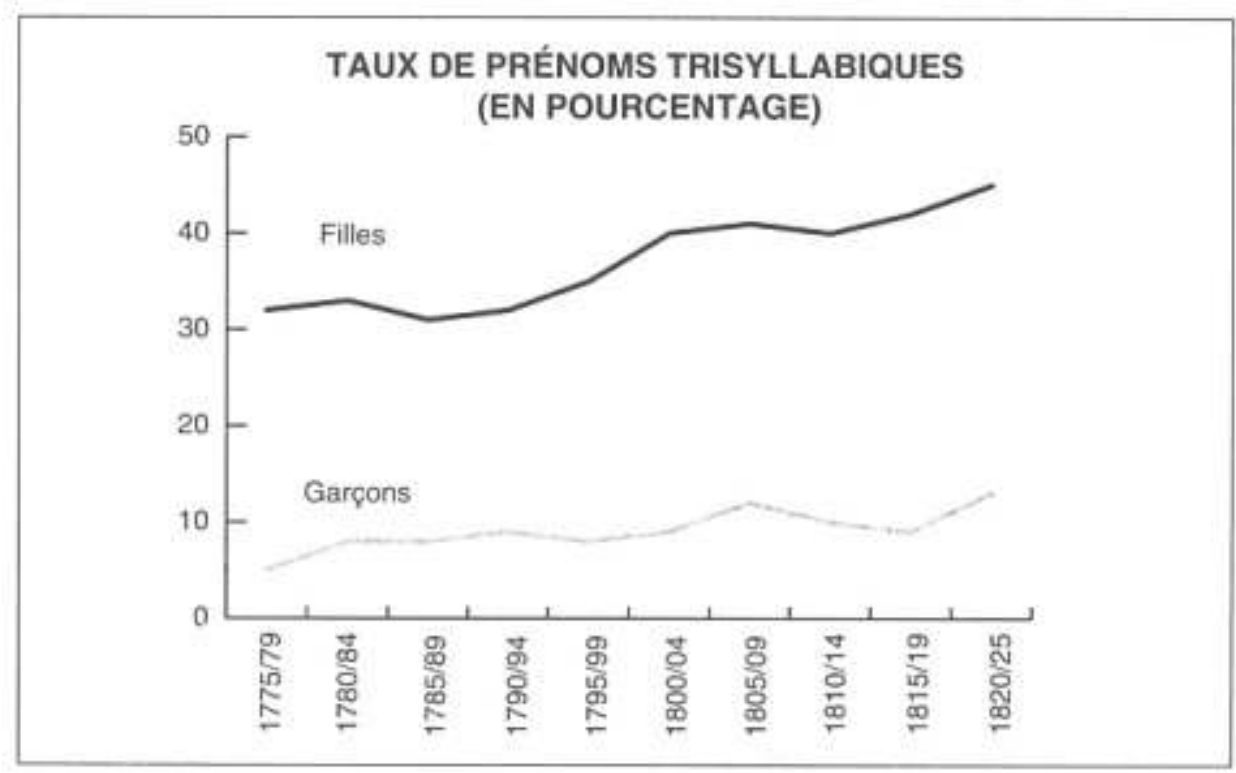

L'écart s'explique partiellement par l'existence de prénoms féminins composés à partir d'un prénom masculin augmenté d'un suffixe, donc forcément plus long, et par le succès croissant de ce type de prénoms. Mais une telle évolution exige non seulement l'acceptation de la complication relative que représente l'allongement, mais aussi l'adhésion à un goût nouveau pour les formes plus complexes et plus esthétisantes.

De manière très logique, l'allongement des prénoms féminins s'accompagne d'un progrès important des terminaisons en ie et en ine. Elles représentent (Marie non inclus) $11 \%$ des prénoms attribués en $1775 / 1779$ et $29 \%$ en 1820/1825 (graphique ci-dessous). Si le phénomène est en partie lié à l'apparition de nouveaux prénoms féminins dérivés de prénoms masculins, il révèle également le succès d'une sonorité spécifiquement féminine, peut-être considérée désormais comme un attribut de la féminité.

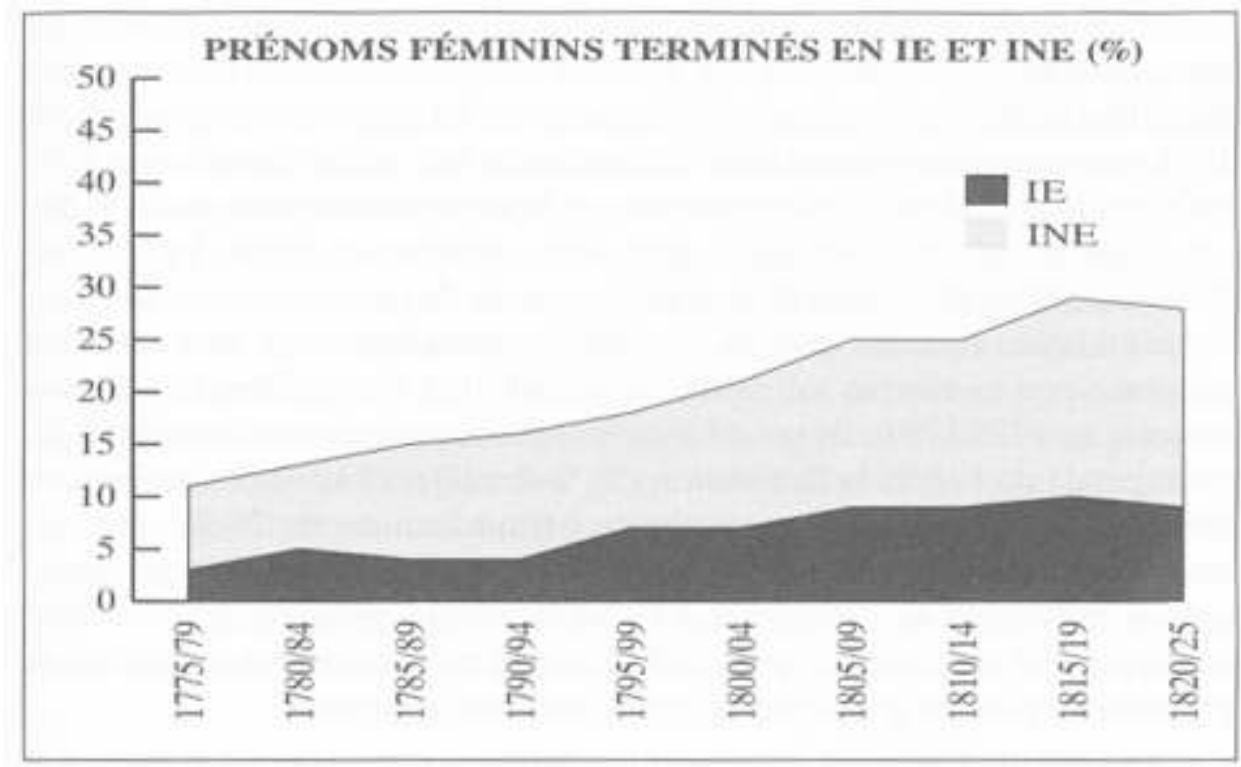

Le nombre de prénoms

C'est la seule donnée relativement stable sur l'ensemble de la période. Le graphique cidessous montre une moyenne variant faiblement autour de deux prénoms par enfant, 
en légère hausse de 1775 à 1805, puis en léger recul par la suite. Bien qu'apparemment faible, l'amplitude observée (de 2,06 à 2,20) n'est pas négligeable pour une moyenne et indique une évolution qui mérite d'être analysée.

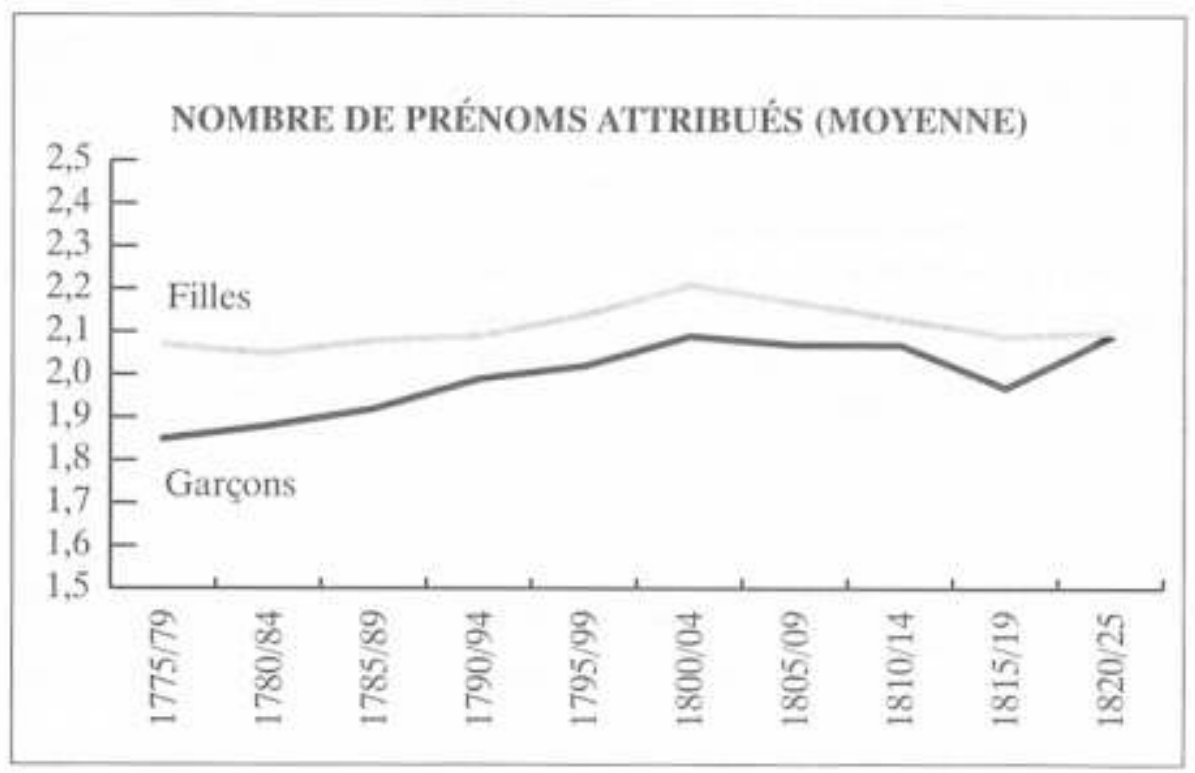

L'étude du nombre d'enfants ayant reçu un, deux, trois prénoms ou plus, en fournit l'explication. Le prénom unique reste une pratique très minoritaire, plus encore pour les filles que pour les garçons, stable autour de $10 \%$ des naissances tout au long de la période. Les enfants ayant reçu deux prénoms sont de loin les plus nombreux ils représentent plus de $70 \%$ des naissances avant la Révolution, puis leur importance faiblit légèrement (65\% en 1800-1804) avant de se stabiliser en fin de période autour de $70 \%$. Cette variation est liée à celle du nombre d'enfants ayant reçu trois prénoms ou plus peu nombreux en début de période (14\% des filles et 6\% des garçons en 1775-1779), ils voient leur importance augmenter considérablement pendant et après la Révolution (27\% des filles et $18 \%$ des garçons en 1800-1804), puis se stabiliser en fin de période autour de $20 \%$ des naissances pour les deux sexes. Le décalage est constant entre les prénoms féminins et masculins, même s'il tend à s'atténuer progressivement les filles reçoivent plus rarement un prénom unique, et nettement plus souvent trois prénoms ou plus, ce qui explique l'écart entre les moyennes.

Les valeurs observées ici ne sont pas surprenantes elles confirment des tendances générales déjà mises en évidence pour des régions proches de Paris ${ }^{3}$. Le passage du prénom unique aux prénominations multiples est massivement réalisé en Île-de-France dès la première moitié du xviIIesiècle, et les prénominations triples ou quadruples existaient également bien avant 1775. La période étudiée ici voit simplement croître leur importance, une évolution amorcée longtemps auparavant, que la Révolution stimule temporairement. Le principal élément nouveau est finalement l'élargissement social de cette pratique. Avant la Révolution, les enfants recevant trois prénoms ou plus appartenaient essentiellement aux classes les plus aisées. À la fin de la période étudiée, leur répartition entre les différentes catégories socioprofessionnelles correspond quasiment à celle de l'ensemble des naissances.

21 Comment expliquer l'influence modeste de la période révolutionnaire sur des évolutions visiblement inscrites dans la longue durée, et dont les causes paraissent avant tout structurelles Dans son enquête sur le corpus falaisien, Jean-Pierre Lethuillier 
montre le rapport direct et permanent entre l'évolution du corpus et celle du nombre de prénoms attribué aux enfants ${ }^{4}$. Si le besoin d'individualisation, très fort au xviIIesiècle, ne peut plus être satisfait que par la multiplication des prénoms lorsque le corpus est devenu insuffisant, à l'inverse l'accroissement du stock répond d'une autre manière à cette exigence et rend inutile un allongement des prénominations. Cette hypothèse est ici en grande partie vérifiée dans une période marquée par l'accroissement continu du corpus, le nombre de prénoms attribués ne progresse que faiblement. De même, la poussée des prénominations triples ou quadruples, dans la décennie qui suit la Révolution, se produit dans la seule période où le corpus régresse légèrement, du fait de la disparition des prénoms républicains et du refus par le clergé des prénoms trop innovants. Enfin, le nombre de prénoms attribués tend à augmenter davantage pour les garçons, dont le stock de prénoms progresse plus modérément. Cette explication a cependant ses limites la hausse tendancielle - même modeste - du nombre de prénoms attribués, la progression sensible des prénominations triples ou quadruples, montrent l'existence d'autres facteurs d'évolution, concernant notamment les critères de choix.

Systèmes de références : plus de liberté ?Marie et autres saintes

22 Avant la Révolution, six prénoms représentent à eux seuls près de $60 \%$ de l'ensemble des prénoms attribués. Marie, avec plus de 30\% à lui seul, joue un rôle particulier $65 \%$ des filles reçoivent ce prénom, presque toujours en première place. Même s'il ne s'agit pas d'une manifestation directe du culte marial mais plutôt, comme le suggère BenoîtGarnot, une simple "habitude socialisées, son inspiration religieuse semble néanmoins indiscutable. La disparition fréquente de Marie dans les mentions secondaires des registres paroissiaux (marraines, témoins des mariages) et dans les archives notariales et judiciaires, montre qu'il est peu utilisé comme prénom usuel, ce qui confirme le caractère quasi rituel de ce choix. Viennent ensuite Anne, Geneviève et Marguerite, saintes dont le culte est très vivant dans la région, Louise et Françoise, dont le succès semble lié à celui de Louis et François, deux prénoms parmi les plus fréquemment attribués aux garçons.

À partir de la Révolution, le recul de tous ces prénoms est rapide et constant. Marie connaît le plus spectaculaire, tombant en cinquante ans de $31 \%$ à $12 \%$ des prénoms attribués (voir annexe 2). En 1820-1825, seulement 25\% des filles reçoivent ce prénom, et la fréquence de son placement en deuxième ou troisième position montre qu'il tend à devenir de plus en plus un prénom comme les autres. Louise et Françoise se maintiennent, ce dernier prénom sans doute stimulé par sa reconversion en prénom à connotation patriotique à partir de la Révolution. Mais les trois autres saintes traditionnelles connaissent également un recul spectaculaire Anne, Geneviève et Marguerite, qui représentaient ensemble 18\% des prénoms attribués en 1775-1779, tombent à moins de $7 \%$ en 1820-1825, et disparaissent de la liste des six prénoms les plus utilisés. Ce recul s'inscrit dans un mouvement général de diversification des choix, puisque dans le même temps l'importance des dix prénoms les plus attribués tombe de $71 \%$ à $46 \%$, celle des six prénoms les plus utilisés de $60 \%$ à $37 \%$. Mais ce n'est certainement pas un hasard si les prénoms à forte connotation religieuse sont les plus touchés.

24 L'influence de la Révolution est ici déterminante. On invoquera bien sûr en premier lieu le recul de l'emprise religieuse sur la vie familiale, attestée par de nombreux autres indicateurs, et qui détermine la même désaffection pour certains prénoms masculins, 
bien que celle-ci soit sensiblement moins marquée Jean, Baptiste, Toussaint, les saints évangélistes, les saints patrons des paroisses ou des corps de métiers. Mais d'autres évolutions viennent accélérer le processus augmentation d'ensemble du stock des prénoms, importance accrue des phénomènes de mode, succès de prénoms mis en valeur par le contexte politique ou idéologique, la vie culturelle et artistique. Le renouvellement des critères de choix ne pouvait s'opérer qu'au détriment de la religion.

Le contexte politique et culturel

La période connaît très peu d'emprunts directs de prénoms féminins à des personnages contemporains. Souverains et leaders politiques étant essentiellement des hommes, les possibilités dans ce domaine étaient de toute façon bien réduites. Les prénoms des reines et impératrices n'ont suscité aucun engouement entre 1775 et 1825 mais ont connu, bien que dans des proportions modestes, des évolutions significatives.

Antoinette est un prénom rare en début de période (0,7\% des attributions en 1775-1779), ce qui confirme l'impopularité de la reine jusqu'à la Révolution. Il disparaît en 1793-1794, puis réapparait et progresse ensuite régulièrement, jusqu'à représenter en 1820-1825 environ $2 \%$ des prénoms attribués (voir annexe 2 ) : une hausse modeste mais bien réelle, à rapprocher de celle de Louis et Louise, et dont l'interprétation est malaisée. Il paraît difficile d'y voir une manifestation délibérée d'attachement à la monarchie. A contrario, on peut envisager que la disparition du couple royal et l'abolition de la monarchie aient levé toute ambiguïté dans ce domaine, et ainsi permis d'attribuer ces prénoms plus facilement, sans aucune crainte ni arrière-pensée.

27 Joséphine connaît une évolution plus originale (voir annexe 2) inexistant lui aussi avant la Révolution ( $0,2 \%$ des prénoms attribués en 1775-1779), il connaît ensuite une progression constante jusqu'à la fin de la période. La forte accélération de cette hausse en 1805-1809, c'est-à-dire entre le sacre et la répudiation de l'impératrice, révèle une influence qui n'est pas forcément la preuve d'une adhésion politique, surtout lorsqu'on connaît la forte impopularité de l'empereur dans les campagnes d'île-de-France au même moment. Si on peut admettre que la notoriété du personnage a contribué à faire connaître le prénom, son succès a survécu au règne de l'éphémère impératrice, probablement en relation avec la vogue des prénoms en ine.

28 L'influence du contexte politique, et en particulier de la Révolution, marque le corpus des prénoms féminins sous des formes plus originales que le simple emprunt à des personnages contemporains. Ainsi, la diffusion de nouvelles références idéologiques suscite un afflux de nouveautés prénoms républicains bien sûr, mais aussi emprunts à la mythologie ou à des personnages de l'histoire antique (Aglaé, Thaïse, Judith), souvent choisis pour leur valeur symbolique. Le phénomène est toutefois nettement moins important pour les filles que pour les garçons, et reste très marginal, même en l'anII. Le changement de sens de certains prénoms, prenant momentanément une connotation patriotique, reste lui aussi très limité Victoire est périodiquement stimulé par les campagnes militaires de la Révolution et de l'Empire, et régresse à nouveau à partir de 1805-1809 (voir annexe 2) Françoise se maintient à partir de la Révolution beaucoup mieux que les autres prénoms traditionnels, sans doute grâce à ce changement de signification. Mais dans les deux cas, s'agissant de prénoms anciens, il est impossible d'évaluer les rôles respectifs du changement et de la continuité.

29 L'influence de la production littéraire et artistique prend à partir de la Révolution une ampleur qui est en elle-même un élément nouveau. De nombreux prénoms féminins 
sont empruntés à des héroïnes de romans (Clarisse, Héloïse, Virginie), de théâtre (Athenaïse, Nanette, Rosine, Zaire, Zulma) ou d'œuvres musicales, elles-mêmes souvent adaptées de la littérature (Anaïs, Euphrosine, Zoé, Zelia). Le même phénomène est bien mis en évidence par AlainCroix et Pascalescilbo dans la région nantaise ${ }^{6}$. C'est la multiplicité de ces prénoms, plus que le nombre d'occurrences de chacun d'eux, le plus souvent bien modeste, qui marque l'ampleur du mouvement, cette fois-ci beaucoup plus important pour les filles que pour les garçons. Enfin, son élargissement à toutes les classes sociales et à tous les milieux géographiques pose le problème du mode de transmission pour qu'un jardinier ou un voiturier d'Ormesson ou d'Aulnay donne à sa fille le prénom de l'héroïne d'une pièce de théâtre jouée à Paris, qu'il n'a probablement pas vue lui-même, il faut qu'il en ait entendu parler ou qu'il connaisse quelqu'un ayant donné ou reçu ce prénom. Cela implique soit une large diffusion, écrite ou orale, de l'information sur la production littéraire et artistique, soit un élargissement des relations entre les classes sociales, soit les deux, et dans tous les cas la levée de barrières mentales et d'inhibitions rendant possible une telle liberté d'expression. Le prénom Virginie, ayant connu un succès largement supérieur à tous ceux de la catégorie (121 occurrences entre 1790 et 1825, voir annexe2), permet deux observations probantes : dans $87 \%$ des cas, il est donné comme deuxième ou troisième prénom, et dans $72 \%$ des cas il n'est emprunté ni à la mère, ni à la marraine de l'enfant. Peut-être fournit-il ainsi un exemple très caractéristique d'un type de prénoms choisis par goût, par attrait de la nouveauté ou sous l'influence de la mode, qui viennent s'ajouter aux prénoms transmis par les parents ou les parrains mais ne les remplacent que partiellement. L'apparition de la nouveauté se concilie avec le maintien de la tradition.

Parents et parrains

La transmission des prénoms par les parents et les parrains était avant la Révolution un modèle quasiment exclusif, auquel n'échappaient que 3\% des enfants. Entre 1775 et 1825, cette situation se modifie sensiblement. L'emprunt des prénoms aux parents recule constamment, en particulier pour les filles (55\% en $1775-1779,49 \%$ en 1820-1825). En revanche, la transmission par le parrain et la marraine reste importante, et concerne tout au long de la période $90 \%$ des enfants des deux sexes. On observe enfin une forte progression des prénoms n'appartenant ni aux parents, ni aux parrains (graphique ci-dessous) de $24 \%$ à $40 \%$ d'enfants concernés, de $24 \%$ à $43 \%$ des filles entre 1775-1779 et 1820-1825. En fin de période, $8 \%$ des enfants et $9 \%$ des filles reçoivent des prénoms totalement étrangers à ceux de leurs parents et parrains. 


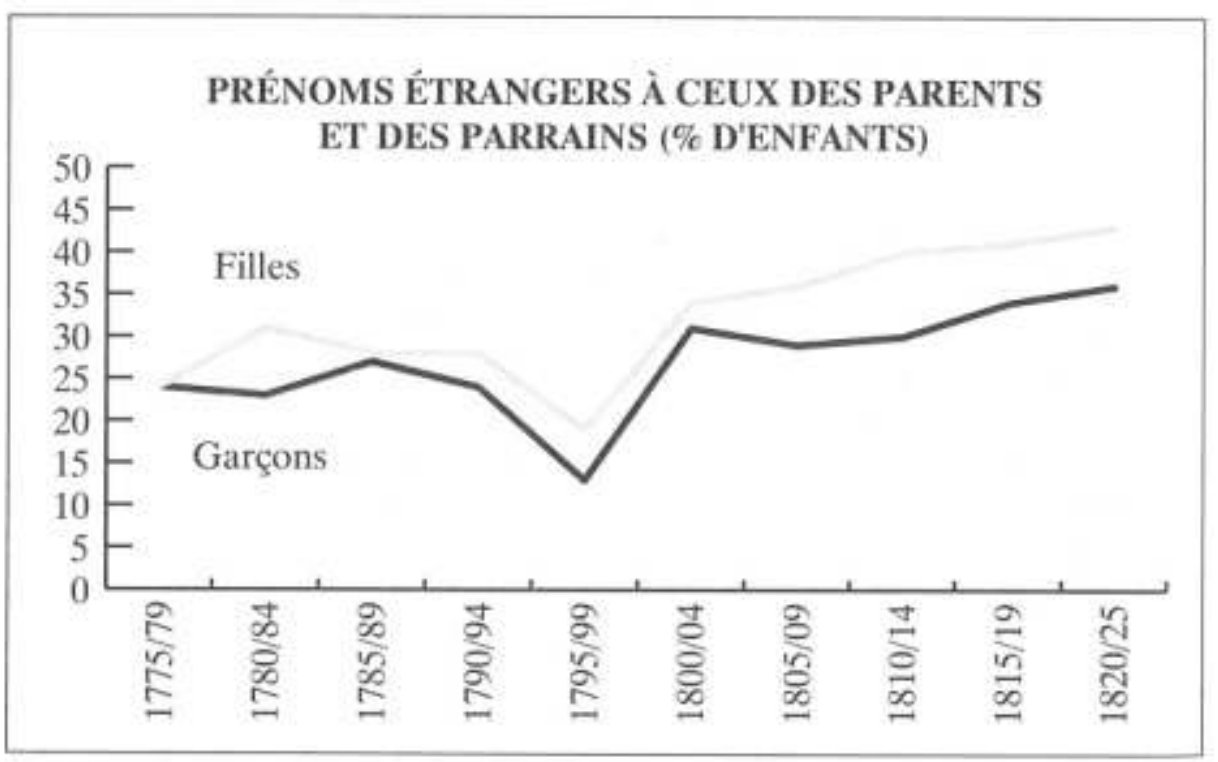

L'étude des associations de prénoms met en évidence des "formules de prénomination particulièrement fréquentes. L'association de deux prénoms empruntés à la mère et à la marraine pour les filles, au père et au parrain pour les garçons, est la formule la plus répandue avant la Révolution (41\% des filles et 36\% des garçons en 1775-1779). C'est, avec les formules à un seul prénom, celle qui enregistre en cinquante ans le recul le plus important $10 \%$ environ pour chacun des deux sexes. La formule comportant deux prénoms empruntés au parrain et à la marraine était également très utilisée avant la Révolution, surtout pour les filles (24\% des garçons, $34 \%$ des filles en $1775-1779$ ). Elle garde toute son importance pendant et après la Révolution, avec un rééquilibrage entre les deux sexes en fin de période autour de $30 \%$. Mais l'évolution la plus spectaculaire est la multiplication des formules comprenant des prénoms hors références, qu'elles soient à deux prénoms (de $11 \%$ à $26 \%$ pour les filles, de $14 \%$ à $28 \%$ pour les garçons), ou à trois prénoms (de $6 \%$ à $15 \%$ pour les filles, de $5 \%$ à $15 \%$ pour les garçons).

L'étude mériterait d'être affinée en dissociant les aînés des autres enfants. Elle exige un important travail de reconstitution des familles, qui n'a pu encore être mené à bien. Compte tenu de la baisse sensible de la fécondité des couples durant les cinquante années étudiées, les statistiques brutes tendent à augmenter l'importance des aînés dans l'effectif global des naissances. Or, on sait que l'attribution d'au moins un prénom transmis par les parents a toujours été plus fréquente chez les aînés, notamment les garçons. Les variations observées à ce sujet n'en sont donc que plus significatives.

Bien que les écarts soient ici peu marqués entre les deux sexes et tendent à s'atténuer tout au long de la période, on constate que les filles, plus concernées avant la Révolution par les deux formules les plus répandues (deux prénoms, empruntés soit à la mère et à la marraine, soit au parrain et à la marraine), sont plus fortement touchées par l'afflux des prénoms hors références, le plus souvent rajoutés aux emprunts traditionnels. On ne peut écarter l'hypothèse, non quantifiable ici faute d'informations, d'un apport accru de prénoms hérités des grands-parents. Mais le nombre important de prénoms nouveaux parmi ces rajouts accrédite l'idée d'un recul des modes de transmission traditionnels au profit de critères de choix plus libres et plus personnels, où le goût peut jouer un rôle non négligeable. La tendance paraît nettement plus marquée pour les filles que pour les garçons. Dans cette évolution continue, on constate 
que la période révolutionnaire ne marque ni cassure, ni accélération du processus, à l'exception d'une légère hausse du nombre d'enfants ayant reçu des prénoms entièrement hors références, ce qui s'explique en grande partie par la vague éphémère des prénoms révolutionnaires.

Les effets directs de la RévolutionPrénoms révolutionnaires

Les prénoms révolutionnaires ont connu dans les onze villages étudiés un succès supérieur à celui de la plupart des autres régions de France, mais néanmoins très limité dans le temps 2 enfants concernés durant les premiers mois de 1793, 62 en l'an II (soit $23 \%$ des naissances), 2 en l'anIII, et 4 durant les trente années suivantes.

L'écart entre les deux sexes est ici très important, puisque seulement $14 \%$ des filles reçoivent des prénoms révolutionnaires en l'an II, contre $32 \%$ des garçons. Mais le décalage est également qualitatif. Parmi les enfants ayant reçu des prénoms révolutionnaires, $18 \%$ des filles ont exclusivement ces prénoms, contre $38 \%$ des garçons. De même, les prénoms à forte connotation militante (héros de la Révolution, concepts idéologiques, personnages de référence) sont beaucoup plus attribués aux garçons (80\%) qu'aux filles (20\%). Il est vrai que ces personnages sont essentiellement de sexe masculin... Pour les prénoms de simple "sensibilité révolutionnaire (jours et mois du calendrier républicain, mythologie et antiquité, valeurs morales), l'écart est moins important $70 \%$ sont attribués aux garçons et 30\% aux filles, celles-ci étant fortement présentes dans les prénoms empruntés au calendrier républicain (voir annexe3).

Une étude mois par mois sur l'an II (graphiques ci-après) met en évidence deux périodes. La première, de brumaire à ventôse, semble correspondre à une phase de politisation dynamique. Les prénominations entièrement révolutionnaires y sont plus nombreuses, de même que les prénoms les plus militants. Dans $62 \%$ des cas, les prénoms républicains sont placés en première position. Les filles y sont beaucoup moins présentes que les garçons. La deuxième période, de floréal à fructidor, présente une typologie entièrement différente les prénominations entièrement révolutionnaires se raréfient, les prénoms militants deviennent fortement minoritaires, enfin les prénoms républicains sont beaucoup plus rarement en première position (17\% des cas). Les filles y sont plus fortement représentées, à tel point que de prairial à thermidor, elles reçoivent plus souvent des prénoms révolutionnaires que les garçons.
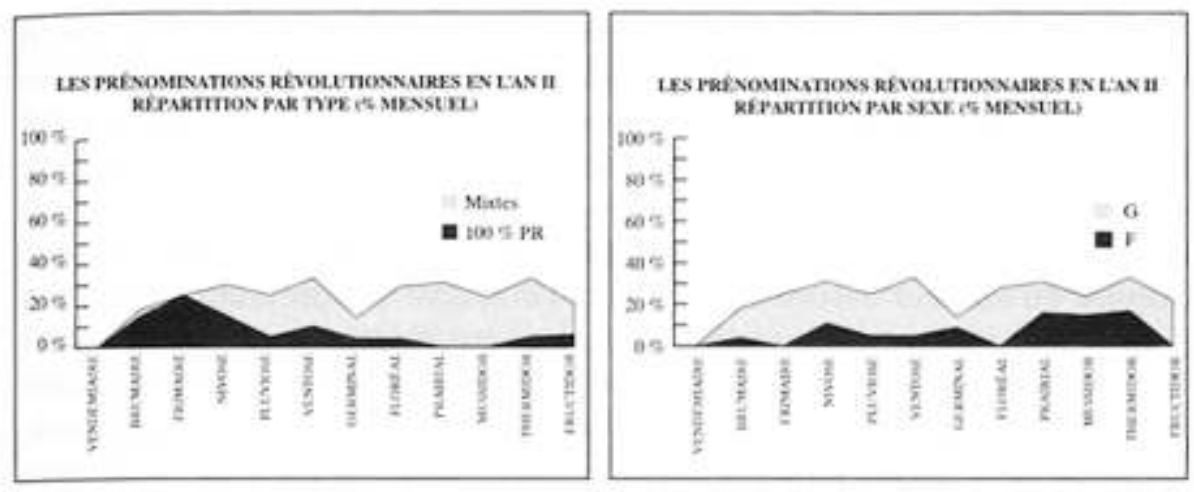

La différence entre les deux périodes s'explique aisément par leur contexte l'une marquée par une forte radicalisation politique, l'autre par une situation plus tendue et incertaine, suscitant sans doute des attitudes plus prudentes. Les écarts quantitatifs et 
qualitatifs entre les deux sexes semblent étroitement liés les uns aux autres, et révèlent des sensibilités très différentes aux garçons des prénoms plus fortement politisés, témoignant d'une plus forte implication dans la vie publique, aux filles des prénoms politiquement plus neutres, relevant davantage de choix culturels ou esthétiques. Le prénom marque ainsi, indirectement, la place de chacun dans la société. En règle générale, les prénoms révolutionnaires ne se transmettent pas. Un seul cas, relevé à Gennevilliers, livre des détails intéressants. Le prénom Argile est attribué à deux filles du village, une en l'an XIII et l'autre en 1820. La première, prénommée Geneviève Argile Henriette, non baptisée, est la cousine d'une enfant née en l'an II et prénommée Argile Égalité. La seconde, prénommée Argile Delphine, sœur de la précédente, est baptisée avec pour marraine la même cousine. Le prénom a été accepté au baptême, et même en première position. Mais sur l'acte, la marraine n'a plus qu'un seul prénom Argile. La disparition du prénom Égalité peut être due à un refus d'inscription par le curé, témoignant des limites de la tolérance de l'Église en la matière, à moins qu'il ait été abandonné par l'intéressée elle-même.

Le baptême contre l'état civil

L'absence de transmission des prénoms républicains, comme leur très faible taux de conservation après la Révolution, ont été grandement facilités par la prédominance des prénominations mixtes, mais aussi par le fait que de nombreux enfants ont reçu au baptême des prénoms différents de ceux de l'état civil. À l'origine, le phénomène est directement lié à celui des prénoms révolutionnaires, bien évidemment refusés par l'Église. Pour les baptêmes clandestins de l'anII comme pour les "rattrapages de baptêmes à la reprise du culte en l'anIII, les parents ont donné d'autres prénoms que ceux de l'état civil. Si au départ l'Église a sans doute imposé les suppressions ou remplacements de prénoms non admis, ainsi que les normalisations orthographiques, la formulation des actes indique souvent que les familles sont bien à l'initiative de la multiplication des doubles prénominations. Il faut toutefois être très prudent quant à l'interprétation de la rédaction des actes si ceux de l'état civil indiquent souvent que les prénoms ont été donnés par le père et ceux du baptême par le parrain ou la marraine, il peut s'agir d'une simple formule rituelle, n'excluant pas l'hypothèse d'un choix concerté, répondant à des critères plus complexes. Cependant, l'idée semble acquise qu'on peut désormais donner à l'état civil des prénoms choisis par goût et au baptême des prénoms répondant aux exigences de l'Église et des traditions familiales un exemple parfait de la coexistence, très caractéristique de la période, entre contrainte et liberté, changement et continuité. L'évolution est rapide (graphique ci-dessous) de $7 \%$ à $12 \%$ des naissances entre 1792 et 1825 . Elle est plus forte et plus régulière pour les filles (de $5 \%$ à 14\%), moins touchées que les garçons par les corrections liées aux prénoms de l'anII. 


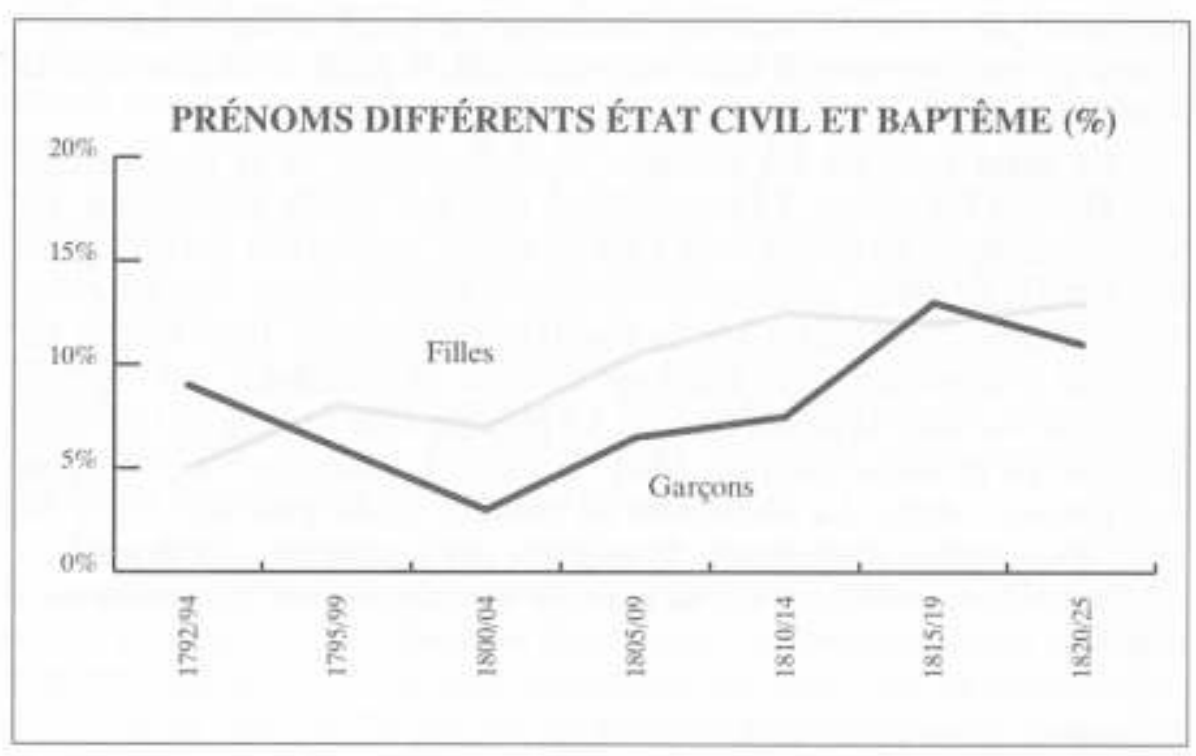

Parmi les 561 cas recensés entre 1792 et 1825 dominent trois types de situations les suppressions ou remplacements au baptême de prénoms non admis par l'Église, les normalisations orthographiques, les ajouts de prénoms empruntés au parrain ou à la marraine en plus ou à la place de prénoms de l'état civil. Si le premier cas de figure ne concerne les garçons que pour les années 1792-1794, en raison du refus par l'Église des prénoms révolutionnaires, il touche les filles de manière constante jusqu'en 1825, la plupart des nouveaux prénoms étant considérés d'emblée comme étrangers au martyrologe chrétien, même lorsqu'ils dérivent de noms de saints.

Les normalisations orthographiques sont un autre moyen de régler le problème Lussine à l'état civil devient Luce au baptême, Adelina devient Adèle, Jenny devient Eugénie, Héloïse devient Louise, Athénaïse devient Adélaïde... On relève de sensibles différences d'une paroisse à l'autre, l'attitude du curé étant déterminante en la matière, mais aussi une atténuation progressive du conflit au fil des années. Il semble que l'Église, soucieuse de rétablir avant tout la pratique des sacrements et la fréquentation du catéchisme, ait accepté de faire des concessions dans des domaines jugés moins prioritaires, tels que le respect des simples préceptes ou le choix des prénoms. Ainsi, Héloïse, Florentine ou Élisa, systématiquement éliminés au lendemain de la Révolution, sont acceptés en 1820-1825 dans la plupart des paroisses.

L'ajout de prénoms empruntés aux parrains ou aux marraines est de loin la situation la plus fréquente ( $46 \%$ des cas chez les filles, $56 \%$ chez les garçons). Son importance est en augmentation constante jusqu'à la fin de la période. Elle témoigne d'un souci croissant de concilier le libre choix des prénoms avec les contraintes des relations sociales. Cette pratique est parfaitement acceptée par l'Église, puisque certains actes de baptême y font clairement allusion, l'un d'eux précisant même que le changement apporté est "sans conséquence ${ }^{7}$...

Une question essentielle vient naturellement à l'esprit quels prénoms l'enfant porterat-il ensuite Ceux de l'état civil ou ceux du baptême Une recherche sur le suivi des prénoms révolutionnaires montre que, dans la quasi-totalité des cas, ceux-ci ont été abandonnés à l'âge adulte, au profit de prénoms traditionnels associés ou, à défaut, de prénoms de baptême. Chez les filles, cet abandon est systématique. Nous n'en donnerons qu'un exemple, celui d'Anne Cloriande Converset, née à Chennevières en l'an II 
et baptisée clandestinement Anne Henriette ${ }^{8}$. Lors de son mariage civil en 1814, elle refuse ses prénoms d'état civil et signe Henriette. La rédaction de l'acte donne lieu à un festival de ratures, qui témoigne d'un certain malaise ${ }^{9}$. Trois ans plus tard, sur la liste de recensement de 1817, elle figure sous l'unique prénom d'Henriette.

L'étude des autres cas de figure exigerait une enquête beaucoup plus lourde, comprenant un important travail de reconstitution des familles, pour identifier avec certitude les centaines de personnes concernées et retrouver dans de multiples sources leur prénom usuel. Elle n'a pas été entreprise ici, d'autant que le devenir des prénoms entre la naissance et l'âge adulte met en cause non seulement le choix des parents, mais également celui de l'enfant lui-même devenu adulte, et répond donc à des règles spécifiques. Il constitue à ce titre une autre direction de recherche.

Nouvelle image, nouveau statut?

Dans un système de prénomination déjà en mouvement, la Révolution apporte des changements importants, témoignant de manière indiscutable de l'émergence de nouvelles conceptions des relations familiales. Si les modes de transmission traditionnels subsistent encore, ils ne sont plus obligatoires. Le choix des prénoms laisse de plus en plus de place à la diversité, à la nouveauté, à l'originalité, à l'expression du goût et à des références autres que religieuses. Même si ces tendances étaient déjà perceptibles -bien que de manière très modeste - au cours du XviIIesiècle, seules les classes sociales les plus favorisées se permettaient alors de prendre quelques libertés à l'égard des traditions. L'apport essentiel de la Révolution est l'élargissement à tous les milieux et à toutes les classes sociales de pratiques auparavant réservées à une élite. Le contexte politique et idéologique, la vague des prénoms révolutionnaires en l'anII, mais plus encore le relâchement de l'emprise religieuse sur la vie des familles, ont favorisé ces évolutions. Perceptible à travers de multiples indicateurs, une nouvelle période d'accélération des changements vers 1805-1815, à l'arrivée à l'âge adulte des enfants de l'anII, peut être également considérée comme un effet à retardement de la Révolution.

De toute évidence, ce "vent de liberté souffle plus fortement pour les filles que pour les garçons l'augmentation du stock, l'afflux de nouveaux prénoms, un recul plus marqué des prénoms traditionnels et des anciennes règles de transmission, révèlent à la fois une volonté plus affirmée d'individualisation de l'enfant et un certain détachement des contraintes sociales. Dans le cadre de ces évolutions s'exprime clairement une volonté de marquer davantage à l'aide des prénoms la différence des sexes, comme en témoignent la disparition des prénoms masculins dans le corpus des prénoms féminins, la tendance à l'allongement des prénoms ou la mode de certaines terminaisons, formes esthétiques peut-être de plus en plus considérées comme des attributs de la féminité.

Cette reconnaissance implicite d'une spécificité féminine, marquée dès la naissance par le choix des prénoms, paraît témoigner d'un intérêt accru pour les enfants de sexe féminin, et par là même d'une évolution de l'image des filles dans un sens plus égalitaire. L'idée exigerait cependant d'être confirmée par d'autres indicateurs.

Peut-on aller plus loin dans l'interprétation Il serait tentant d'observer, à partir des différences marquées entre la typologie, les modes d'attribution et les références des prénoms féminins et masculins, l'ébauche d'un "statut culturel féminin tendant au confinement des femmes dans un domaine culturellement bien délimité, celui de l'esthétisme et de la gratuité, pour ne pas dire de la futilité, ce qui tendrait à une forme de marginalisation sociale... Ce serait sans doute aller au-delà de ce qu'on peut faire 
dire à un simple indicateur. De plus, même si l'idée correspond à une image de la femme déjà bien présente dans l'idéologie dominante et qui s'affirmera plus nettement encore au xixesiècle, elle est fortement contredite par la réalité de la vie économique et sociale des classes populaires, et plus particulièrement du milieu rural. Il faudrait alors admettre l'existence d'un décalage entre l'évolution rapide et profonde des idées, des représentations et des pratiques culturelles, et celle, beaucoup plus difficile, des rapports sociaux et des conditions de vie. Mais n'est-ce pas justement une des caractéristiques de la période révolutionnaire

\section{ANNEXES}
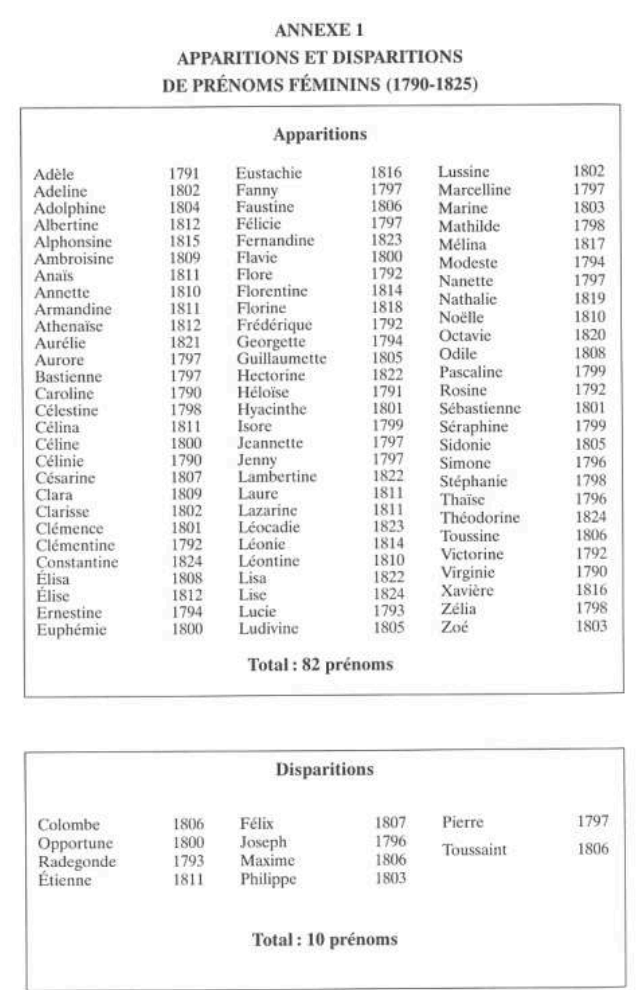
ANNEXE 2

QUELQUES ÉVOLUTIONS SIGNIFICATIVES

DE PRÉNOMS FÉMININS (1775-1825)
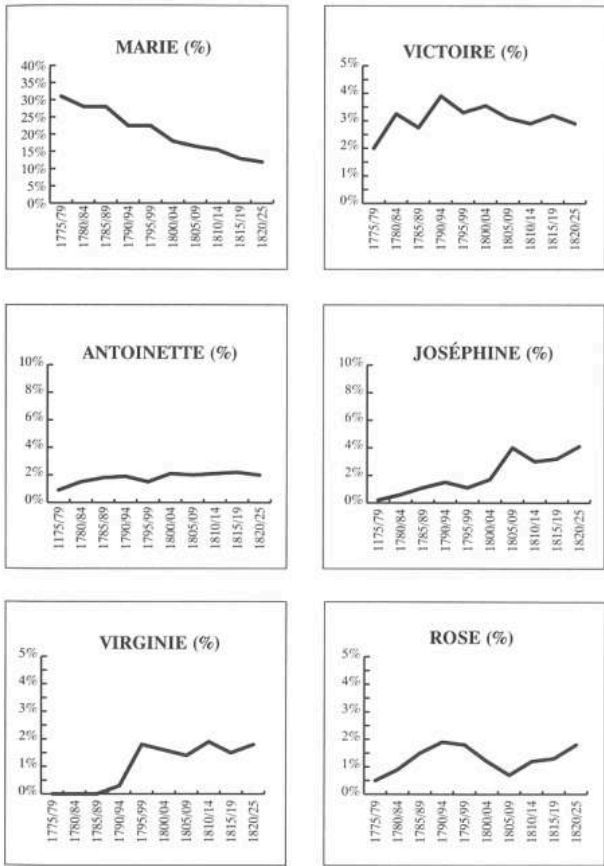

ANNEXE 3

LES FILLES ET LES PRÉNOMS RÉVOLUTIONNAIRES (AN II - AN III)

\begin{tabular}{|c|c|c|}
\hline \multicolumn{3}{|c|}{ CATÉGORIE I } \\
\hline Héros & Valeurs & Références \\
\hline $\begin{array}{l}\text { Le Peleticr } \\
\text { Marat }\end{array}$ & $\begin{array}{c}\text { Égalité } \\
\text { Fraternité } \\
\text { La Montagne } \\
\text { Liberté } \\
\text { Union }\end{array}$ & $\begin{array}{c}\text { Brutus } \\
\text { Corneillia } \\
\text { Franklin } \\
\text { Helvetius } \\
\text { Mucius Scasvola } \\
\text { Rousseasu }\end{array}$ \\
\hline
\end{tabular}

\begin{tabular}{|c|c|c|c|c|}
\hline \multicolumn{5}{|c|}{ CATÉGORIE II } \\
\hline Calendrier $(\mathrm{M})$ & Calendrier (J) & Valeurs & Antiquité & Divers \\
\hline $\begin{array}{c}\text { Floréal } \\
\text { Fructidor } \\
\text { Germinal } \\
\text { Messidor } \\
\text { Octidi } \\
\text { Prairial } \\
\text { Thermidor }\end{array}$ & 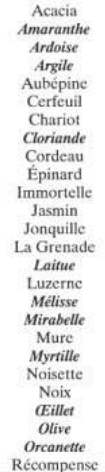 & $\begin{array}{c}\text { Ami } \\
\text { Espérance } \\
\text { Fidele } \\
\text { France } \\
\text { Sage } \\
\text { Sincère }\end{array}$ & $\begin{array}{l}\text { Athènes } \\
\text { Bélisaire } \\
\text { Polynice } \\
\text { Socrate }\end{array}$ & $\begin{array}{l}\text { Désir } \\
\text { Fleur d'épinc } \\
\text { Hysiotrope } \\
\text { Salpêtre }\end{array}$ \\
\hline
\end{tabular}

(Les prénoms attribués à des filles apparaissent en gras et italique)

\section{NOTES}

1.En particulier :-L.PEROUAS (dir.), Léonard, Marie, Jean et les autres, les prénoms en Limousin depuis un millénaire, Paris, 1984, 229 p.-J.-PLETHUiLLIER, "Prénoms et Révolution, enquête 
sur le corpus falaisien, Annales de Normandie, 1989, n 4, pp. 413 à 436.-Ph. DAUMAS, "Prénoms et Révolution, propositions pour une nouvelle approche méthodologique, Revue d'Histoire moderne et contemporaine, 1997, n 1, pp.109 à 132 .

2.Aulnay-sous-Bois, Chennevières-sur-Marne, Gennevilliers, Ivry, Noiseau, Orly, Ormesson, Saint-Rémy-lès-Chevreuse, Sevran, Thiais et Trappes.

3.En particulier :-J.DUPAQUIER, "Prénoms, parrains, parenté. Recherches sur les familles du Vexin français de 1540 à 1900, Mémoires de la société historique et archéologique de Pontoise, du Val d'Oise et du Vexin, T.LXIX, 1980, pp.55-87.-B.GARNOT, “Les prénoms populaires à Chartres au XVIIIesiècle, Revue historique, T. CCLXXVII, 1987, pp.3-10.

4.J.-P.LETHUILliER, op. cit., p. 20.

5.B.GARNOT, op. cit., p.6.

6.A.CRoIX et P.SCILBo (dir.), Brutus, Désir de la Paix et Jonquille : les prénoms de la Révolution en Loire-Inférieure, Nantes, 1993, 143 p.

7.Baptême de Marie Rosalie Lamarche à Ivry, le 3 novembre 1806.

8.Les archives de l'évêché de Versailles conservent pour la paroisse de Chennevières un registre de baptême clandestin, tenu pendant la période d'interdiction du culte, et mentionnant les noms des enfants et de leurs parents, les dates de la naissance et du baptême, les prénoms attribués. Le cas n'est pas exceptionnel.

9.Mariage civil de Jean Baptiste Victor Converset et Anne Cloriande Converset à Chennevières, le 29 décembre 1814.

\section{RÉSUMÉS}

L'étude des prénoms peut être un indicateur culturel pertinent pour la période révolutionnaire, surtout si elle s'intéresse non seulement aux prénoms républicains, mais à l'ensemble du corpus. Ainsi, une série de recherches sur les prénoms féminins, portant sur l'évolution du stock, le nombre, la forme des prénoms, les associations ou les systèmes de références, peut mettre en évidence des changements significatifs.

L'enrichissement et la féminisation du stock, le recul des modes de transmission traditionnels, le renouvellement des références, témoignent d'un "vent de liberté" beaucoup plus fort pour les filles que pour les garçons. L'image des filles semble évoluer dans un sens plus égalitaire, et l'affirmation de formes de prénomination spécifiques témoigne peut-être de la reconnaissance accrue d'une identité féminine, même si son contenu idéologique n'est pas dépourvu d'ambiguïtés.

Girls'Names and their Image : Research into Female First Names in Ile-de-France during the Revolutionary Period (1775-1825)

The study of first names can provide a relevant cultural indicator for the revolutionary period, especially if it covers not just republican forenames, but the corpus as a whole. Thus, a survey of female first names dwelling on the development of the stock, the number and form of forenames, the associations or reference-systems, can shed light on significant changes.

The growth and feminization of the stock, the regression of traditional modes of transmission, the renewal of references reveal that the wind of liberty blows stronger for the girls than for the boys. The image of girls seems to evolve in a more egalitarian fashion, and the affirmation of 
specific forms of naming could well indicate a stronger sense of female identity, even though their ideological content is not without ambiguities.

INDEX

Mots-clés : prénoms, féminité (ou femme), culture

\section{AUTEUR}

PHILIPPE DAUMAS

Université de Rennes 2 